\title{
Observaciones morfométricas y reproductivas en el langostino Pleoticus muelleri Bate, 1888 procedente de embarcaciones comerciales del puerto Rawson, Argentina
}

\author{
Morphometric and reproductive observations of the shrimp Pleoticus muelleri \\ Bate, 1888 from commercial ships off Rawson Port, Argentina \\ Ana E. Ruiz ${ }^{1}$ y Luis F. Mendia ${ }^{1,2}$ \\ ${ }^{1}$ Facultad de Ciencias Naturales, Universidad Nacional de la Patagonia San Juan Bosco, Sede Trelew. \\ Julio A. Roca 115, U9100AQC Trelew, Chubut, Argentina \\ ${ }_{2}^{2}$ Secretaría de Pesca de la Provincia del Chubut. Belgrano 778, 9103, Rawson, Chubut, Argentina \\ anaruiz@ar.inter.net
}

\begin{abstract}
The shrimp Pleoticus muelleri is the most important species in the crustacean fishery off Patagonian Argentine Sea. Morphometric and reproductive aspects of 1,564 specimens from Rawson Port zone, South Argentine, were analyzed. The shrimps were taken approximately $12 \mathrm{~nm}$ by the trawling coastal fleet, between January and April 2001. From the total sample, only $3.9 \%$ were juveniles. Mature and impregnated females were mostly found in January (86.27\%)
\end{abstract}

and the lowest peak was in March. Immature female shrimps were caught in only in April. These results show the importance to characterize the shrimps from landing samples, to the fishery management.

Key words: Crustacea, Patagonian Argentine Sea, reproductive state, morphometry

Su ciclo de vida es corto y es considerado anual para la administración pesquera. La actividad reproductiva se desarrolla a lo largo del litoral patagónico durante la primavera y el verano, con máxima intensidad entre noviembre y marzo. La principal área de desove se sitúa entre los $43^{\circ}$ y $47^{\circ} \mathrm{S}$, en tres zonas principales, frente al puerto Rawson y sectores norte y sur del golfo San Jorge (Boschi 1989, Macchi et al. 1998, Fernández 2005). En este puerto operan 35 embarcaciones desde la costa hasta las $12 \mathrm{mn}$, para buques menores de $21 \mathrm{~m}$ de eslora. La red de pesca empleada para la captura del langostino posee en el copo una luz de malla de $45 \mathrm{~mm}$, lo que hace vulnerables a adultos y juveniles a la pesca comercial (Boschi 1989). En estas embarcaciones no se realizan tareas de clasificación, ya que el total de la captura es desembarcada para su procesamiento en las plantas de pescados y mariscos frescos. Por lo tanto, es de suma importancia la clasificación de los langostinos según su estadio de desarrollo durante las temporadas de pesca, de modo de obtener la información necesaria que permita un mejor manejo del stock pesquero de la zona frente al puerto Rawson. Es así que este estudio clasifica y reconoce, por primera vez, los rasgos morfométricos y aspectos reproductivos en ejemplares de Pleoticus muelleri capturados durante la temporada de pesca en el puerto Rawson. 


\section{Material y métodos}

Se analizó un conjunto de muestras de langostino (Pleoticus muelleri) procedente del área de pesca, ubicada frente al puerto Rawson, área comprendida entre $43^{\circ} 20^{\prime}$ y $43^{\circ} 32^{\prime} \mathrm{S}$ y desde la costa hasta las $12 \mathrm{mn}$, dentro de la Zona de Esfuerzo Pesquero Restringido. Se analizaron 1.564 ejemplares, en catorce muestreos realizados entre enero y abril de 2001, año del máximo desembarque reportado (SAGPyA 2008, Fernández et al. 2007).

Los muestreos fueron realizados dentro de las 15 horas posteriores a la captura, con una frecuencia semanal que estuvo condicionada por la actividad pesquera. La captura fue conservada con hielo y mantenida en cámara de fresco. De un cajón de langostinos, obtenido al azar, de aproximadamente $15 \mathrm{~kg}$, que contiene en promedio 408 individuos, se extrajeron, también al azar, entre 85 y 125 ejemplares para su observación.

Según los criterios metodológicos propuestos por Angelescu \& Boschi (1959), de cada ejemplar se obtuvieron las siguientes medidas morfométricas, consideradas al mm inferior más cercano: Largo total $(\mathrm{Lt})$, Largo caparazón (Lc) y Largo abdomen (La); cada ejemplar fue pesado $(\mathrm{P})$ con precisión de $0,1 \mathrm{~g}$. La distinción entre sexos se realizó por presencia en el macho del órgano petasmal. Se reconocieron los estadios de madurez sexual en hembras para identificar el período reproductivo. Se empleó una escala basada en diferencias de coloración y tamaño de los ovarios. Esta escala incluye tres estadios para las hembras: 1) inmadura cuando el ovario no presenta coloración aparente a través del caparazón, 2) madura cuando el ovario es de un color verde intenso que se trasluce a través del caparazón y puede llegar hasta el último segmento del abdomen, 3) impregnada cuando el ovario se presenta como en el estadio 2 y además en la zona ventral posterior del cefalotórax se encuentra adherido el espermatóforo, que es una pieza única de un color verde claro (Boschi 1989, Iorio et al.1996, Fernández 2005, Castilho et al. 2007).

Se estimaron el promedio, la desviación estándar, la moda y el rango de las medidas morfométricas obtenidas y se obtuvo la distribución de frecuencias de Lc. También, se obtuvo la distribución porcentual mensual de los estadios de madurez del langostino. Además, se determinó el porcentaje de hembras y de machos que no alcanzaron el Lc de primera madurez según el criterio de Iorio et al. (2000), quienes determinaron un Lc de $31 \mathrm{~mm}$ para la primera madurez en hembras y de $28 \mathrm{~mm}$ en machos. Langostinos con Lc menores a estas medias fueron clasificados como juveniles (Bertuche et al. 2000, Fernández et al. 2002).
Los parámetros de las relaciones y entre el largo del caparazón y el peso del langostino (Lc-P) y entre el largo del abdomen y el peso (La-P) fueron estimados con los valores observados para el conjunto de ejemplares inmaduros, con exclusión de los juveniles para descartar diferencias en el crecimiento alométrico relacionadas con los estados de madurez sexual (Díaz et al. 2001, Fernández \& Hernández 2002, Fernández et al. 2002, De la Garza 2003):

$$
\mathrm{P}=\mathrm{a} \cdot \mathrm{Lc}^{\mathrm{b}} ; \quad \mathrm{P}=\mathrm{a} \cdot \mathrm{La}^{\mathrm{b}}
$$

donde, P: Peso (g), Lc: Largo caparazón (mm), La: Largo abdomen (mm), a y b: constantes, obtenidas por mínimos cuadrados.

Previamente, fue aplicado un análisis de regresión para conocer si estas variables de longitud se incrementaron con tasas proporcionales, respecto al Lt. Se aplicó un ANCOVA para determinar si existían diferencias significativas entre los sexos en las relaciones largo-peso estimadas (Díaz et al. 2003).

\section{Resultados}

Para el conjunto de la muestra los promedios fueron: $\overline{\mathrm{Lc}}=$ $39,19 \pm 6,78 \mathrm{~mm}$, (rango: $12-59 \mathrm{~mm}$; moda: $38 \mathrm{~mm}$ ) y $\overline{\mathrm{P}}=36,67$ $\pm 15,03 \mathrm{~g}$ (rango: $3,20-84,70 \mathrm{~g}$; moda: $35 \mathrm{~g}$ ). El rango de Lc varió entre 17 y $59 \mathrm{~mm}$ en las hembras y entre 12 y $48 \mathrm{~mm}$ en los machos. El rango de $\mathrm{P}$ estuvo comprendido entre 3,50 y $84,70 \mathrm{~g}$ en las hembras y entre 3,20 y $51,50 \mathrm{~g}$ en los machos, respectivamente. Las medidas morfométricas presentaron valores medios con tendencia decreciente de enero a abril (Tabla 1).

La distribución de frecuencias de Lc presentó en ambos sexos el intervalo modal principal comprendido entre 39,1 y $42 \mathrm{~mm}$. En las hembras hubo otro secundario entre 48,1 y $51 \mathrm{~mm}$ y en los machos entre 30,1 y $33 \mathrm{~mm}$ (Fig. 1).

En la distribución porcentual mensual de los estadios de madurez sexual se observó, para hembras, que en enero hubo $56,86 \%$ de maduras y $29,41 \%$ de impregnadas. Estos porcentajes fueron decreciendo en los meses siguientes hasta abril, cuando la totalidad de las hembras estaba inmadura (Fig. 2).

La muestra de hembras presentó $2,9 \%$ de juveniles y la de machos, $3,7 \%$. La distribución mensual permitió apreciar que en enero no se registraron juveniles en ninguno de los sexos y que en los meses siguientes, en hembras el porcentaje de los juveniles no superó el $4 \%$. En machos, la proporción de juveniles fue de $6 \%, 11 \%$ y $4 \%$, en febrero, marzo y abril, respectivamente. 
En enero, la cantidad de hembras fue mayor a la de machos. Luego, de febrero a abril se observó una tendencia creciente en la proporción de hembras (Tabla 1). Los ajustes fueron lineales entre Lt-Lc $\left(R^{2} \geq 0,91\right)$ y LtLa $\left(R^{2} \geq 0,92\right)$, condición que indica que estas variables incrementaron con tasas proporcionales.

Las relaciones estimadas entre los largos y el peso presentaron diferencias significativas entre los sexos
$(P<0,05)$ (Fig. 3):

Hembras, $P=0,0037 . \mathrm{Lc}^{2,49}, P=0,0001 . \mathrm{La}^{2,90}$

Machos, $P=0,0022 . \mathrm{Lc}^{2,67}, P=0,0001 . \mathrm{La}^{2,84}$

La relación Lc-P mostró un valor de b significativamente menor en hembras que en machos $(2,49 ; 2,67)$, mientras que en la relación La-P, la constante $\mathrm{b}$ fue superior en hembras $(2,90 ; 2,84)$.

Tabla 1

Promedios, desviaciones estándares, modas y rangos de: Largo total (Lt), Largo caparazón (Lc), Largo abdomen (La), en mm, y Peso (P) en g, del langostino (Pleoticus muelleri). Puerto Rawson, enero-abril 2001

Averages, standard deviations, modes and ranges of: Total length (Lt), Carapace length (Lc), Abdomen length (La), in mm and Weight (P) in g, of shrimp (Pleoticus muelleri). Rawson Port, January-April 2001

\begin{tabular}{|c|c|c|c|c|c|c|c|c|c|}
\hline & & $\mathrm{Lt}$ 오 & $\mathrm{Lt} \sigma^{-1}$ & Lc 오 & Lc $\sigma^{\wedge}$ & $\mathrm{La}$ 오 & La ${ }^{\wedge}$ & P오 & $\mathrm{P} \delta^{-1}$ \\
\hline Enero & Promedio & 187,84 & 162,35 & 50,04 & 39,62 & 89,71 & 81,18 & 62,34 & 40,31 \\
\hline Nㅇ 51 & DS & 8,62 & 5,93 & 3,51 & 1,63 & 4,41 & 3,49 & 8,69 & 4,24 \\
\hline \multirow[t]{2}{*}{ Nó 34} & Moda & 190 & 160 & 48 & 40 & 90 & 80 & 65,7 & 40,4 \\
\hline & Rango & $170-205$ & $150-175$ & $43-59$ & $37-43$ & $80-100$ & $75-90$ & $44,9-82,3$ & $32,4-51,5$ \\
\hline Febrero & Promedio & 156,64 & 141,88 & 41,13 & 34,90 & 75,56 & 72,02 & 39,64 & 29,33 \\
\hline $\mathrm{N}$ 우 481 & DS & 22,92 & 19,19 & 6,73 & 4,87 & 10,96 & 9,63 & 16,47 & 10,23 \\
\hline \multirow[t]{2}{*}{ Nở 263} & Moda & 140 & 160 & 37 & 37 & 70 & 80 & 33,3 & 17,3 \\
\hline & Rango & $65-200$ & $65-170$ & $17-56$ & $15-43$ & $35-110$ & $35-105$ & $3,5-78,9$ & $3,2-47,1$ \\
\hline Marzo & Promedio & 156,04 & 135,38 & 40,79 & 32,74 & 74,86 & 68,90 & 38,81 & 25,12 \\
\hline N우 366 & DS & 19,88 & 19,33 & 5,90 & 5,38 & 9,19 & 9,25 & 14,29 & 9,58 \\
\hline \multirow[t]{2}{*}{ Nở 119} & Moda & 145 & 130 & 37 & 29 & 70 & 65 & 26,7 & 21,3 \\
\hline & Rango & $85-205$ & $75-170$ & 21-58 & $12-48$ & $45-100$ & $40-85$ & $6,8-84,7$ & $3,7-44,4$ \\
\hline Abril & Promedio & 155,70 & 136,90 & 40,55 & 33 & 75,28 & 69,30 & 39,12 & 25,70 \\
\hline N우 179 & DS & 16,79 & 12,32 & 5,08 & 3,21 & 7,66 & 5,69 & 11,30 & 6,55 \\
\hline \multirow[t]{2}{*}{ Nồ 71} & Moda & 155 & 140 & 40 & 34 & 75 & 70 & 38,7 & 25,3 \\
\hline & Rango & $80-205$ & $95-170$ & $20-56$ & $22-41$ & $40-95$ & $50-85$ & $5,9-77,1$ & $9,6-48,9$ \\
\hline
\end{tabular}

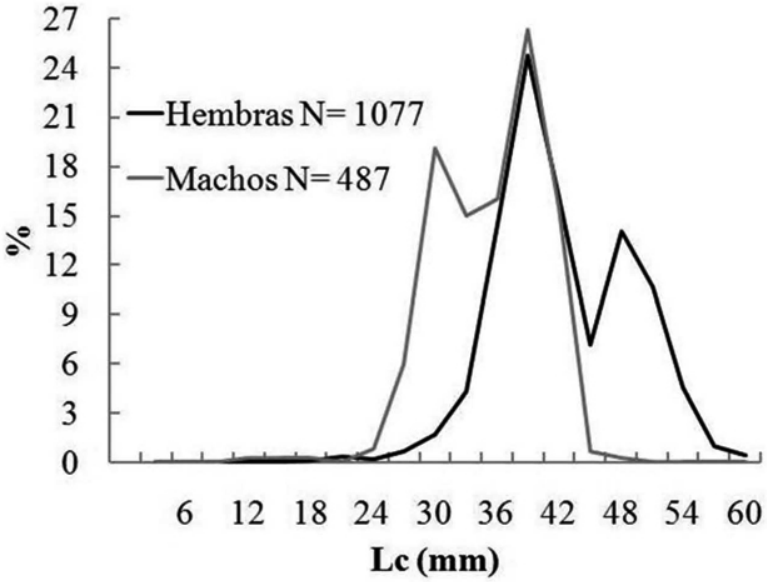

Figura 1

Distribución del largo del caparazón del langostino (Pleoticus muelleri), por sexo. Puerto Rawson, enero-abril 2001

Carapace length distribution of shrimp (Pleoticus muelleri), by sex. Rawson Port, January-April 2001

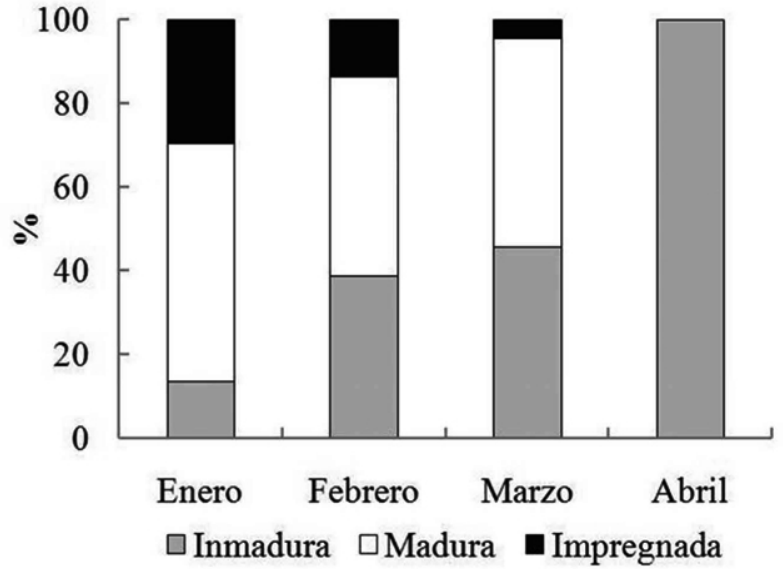

Figura 2

Distribución porcentual mensual de los estadios de madurez sexual en hembras del langostino (Pleoticus muelleri). Puerto Rawson, enero-abril 2001

Monthly percentage distribution of sexual maturity stages in females of shrimp (Pleoticus muelleri). Rawson Port, January-April 2001 

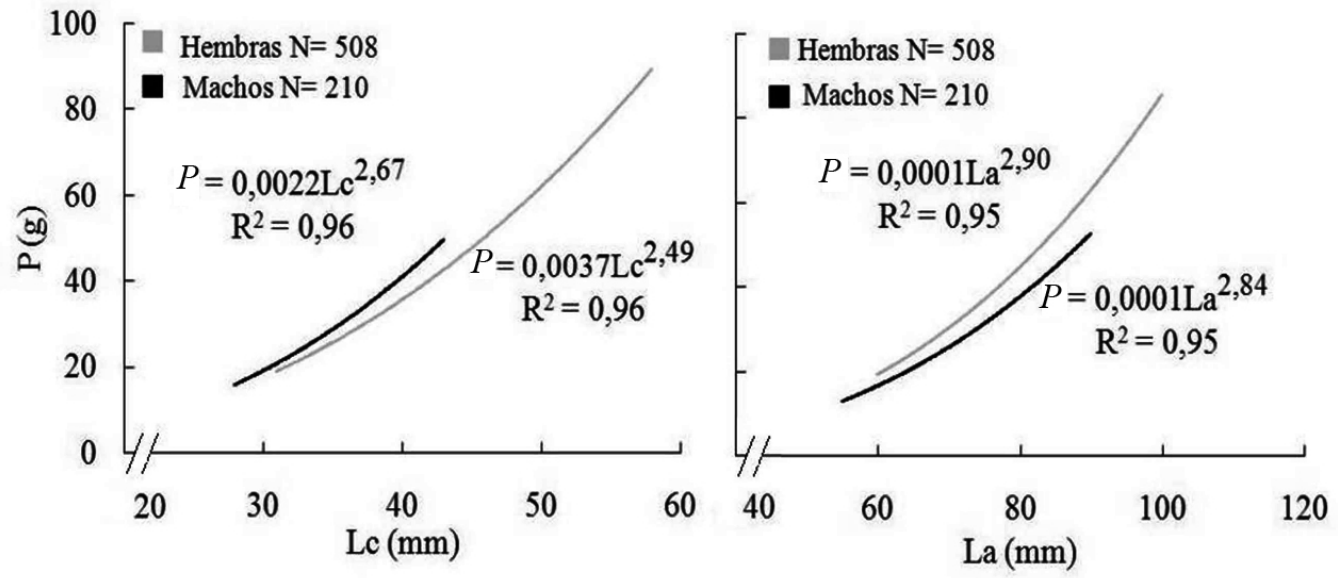

Figura 3

Relaciones largo caparazón-peso y largo abdomen-peso del langostino (Pleoticus muelleri), por sexo. Puerto Rawson, enero-abril 2001

Carapace length-weight and abdomen length-weight relationships of shrimp (Pleoticus muelleri), by sex. Rawson Port, January-April 2001

\section{Discusión}

El conjunto de muestras del langostino Pleoticus muelleri desembarcado por la flota costera que operó desde el puerto Rawson, en temporada de pesca entre enero y abril de 2001, permitió apreciar una distribución porcentual de Lc que es bimodal en ambos sexos, con coincidencia en el intervalo modal principal. En las hembras, el segundo intervalo modal se encuentra desplazado hacia las tallas mayores y en los machos hacia las tallas menores, lo que puede estar relacionado con el dimorfismo sexual característico de esta especie (Angelescu \& Boschi 1959). Se observó presencia mayoritaria de adultos, pues los juveniles en las hembras no superaron el $4 \%$. Como es una especie que migra de sur a norte y que en las zonas de pesca localizadas al sur del golfo San Jorge las capturas están integradas por gran proporción de juveniles (Boschi 1989), estos resultados son importantes para la administración pesquera, ya que muestran que es frente al puerto Rawson donde pueden obtenerse capturas con mayores rendimientos en peso por individuo.

La dinámica del proceso reproductivo del langostino Pleoticus muelleri requiere atención para el manejo de su pesquería, por ser una especie de vida corta (Macchi et al. 1998). La actividad reproductiva se identificó mediante la distribución porcentual mensual de estadios de madurez sexual. En enero se observó el mayor porcentaje de hembras maduras e impregnadas $(86,27 \%)$. Durante los meses siguientes, este porcentaje fue disminuyendo gradualmente hasta abril, cuando la totalidad de las hembras estaban inmaduras.

La escala de madurez sexual utilizada, sustentada en observaciones macroscópicas del ovario, no permitió reconocer entre los ejemplares inmaduros, a los juveniles que maduraban por primera vez. No obstante, como la presencia de hembras juveniles en las muestras de abril fue menor al $4 \%$, se reconoce que la mayor parte de las hembras eran adultas desovadas, lo que permite establecer nuevamente al mes de abril como la fecha de finalización de la actividad reproductiva.

Los resultados relativos a la proporción de sexos en el área de estudio, dentro del lapso observado, permiten apreciar desplazamientos relacionados con la evolución del período reproductivo. Se distingue similar cantidad de machos y hembras en enero, momento de mayor actividad reproductiva y predominio de hembras durante la etapa siguiente cuando decae la madurez sexual. Agrupamientos similares vinculados con el ciclo sexual, fueron señalados para la zona de pesca de Mar del Plata por Angelescu \& Boschi (1959).

Por todos los aspectos señalados y al considerar que en el litoral de Rawson han sido reconocidas para esta 
especie concentraciones de adultos en estado reproductivo entre octubre y diciembre (Fernández 2005), es posible establecer que, en enero, al mismo tiempo que se produjo la mayor actividad madurativa del período estudiado, se inició la declinación reproductiva que alcanzó en abril una etapa de inactividad sexual.

Las relaciones morfométricas entre los largos estudiados (Lc y La) y el peso (P) que fueron obtenidas sólo para los adultos inmaduros, en todos los casos arrojaron para la constante b valores inferiores a 3 , reflejo de alometría negativa. Ello indica que hay un crecimiento más desarrollado en los ejes transversales con un ligero cambio en el espesor del soma, que produce un aspecto de robustez en los ejemplares (Angelescu \& Boschi 1959). En las hembras se reconoció un aspecto de mayor robustez en el abdomen (más aprovechable en la industria de la alimentación) respecto a los machos, con caparazón más esbelto. En un estudio referido a la misma zona, que sólo incluyó la relación Lc-P para hembras obtenidas en noviembre $(\mathrm{N}=70)$, se obtuvo un valor de b similar $(2,42$ según Fernández et al. 2002).

En este estudio se reconoce a la zona del puerto Rawson como un lugar reproductivo para el langostino. Además, el bajo porcentaje de juveniles encontrado, las capturas obtenidas hasta ahora y los desplazamientos espaciales y temporales del stock pesquero documentados en literatura, ubican a esta zona como un adecuado lugar de pesca del langostino. Sin embargo, si algunas condiciones cambian, como el esfuerzo pesquero o las condiciones ambientales, las características biológicas del langostino de la zona del puerto de Rawson podrían verse afectadas. Por lo tanto, es importante tener monitoreos constantes en el tiempo que permitan detectar los cambios biológicos de este recurso, con el propósito de tener la información adecuada y actualizada al momento de tomar decisiones sobre el manejo pesquero del langostino.

\section{Agradecimientos}

Los autores expresan su agradecimiento a la empresa pesquera Interpesca S.A. por proveer las muestras analizadas en este trabajo y permitir el uso de sus instalaciones para realizar las tareas de muestreo. También agradecen a los anónimos evaluadores por las correcciones realizadas.

\section{Literatura citada}

Angelescu V \& EE Boschi. 1959. Estudio biológico pesquero del langostino de Mar del Plata en conexión con la operación nivel medio. Servicio de Hidrografía Naval, Argentina Público H 1017: 1-135.
Bertuche D, C Fischbach, A Roux, M Fernández \& R Piñero. 2000. Langostino (Peoticus muelleri). En: Bezzi SI, R Akselman \& EE Boschi (eds), Síntesis del estado de las pesquerías marítimas argentinas y de la cuenca del Plata. Años 1997-1998, con actualización de 1999. Publicaciones Especiales INIDEP: 179-190.

Boschi EE. 1989. Biología pesquera del langostino del litoral patagónico de Argentina (Pleoticus muelleri). Contribución INIDEP 646: 1-71.

Castilho AL, RC Costa, A Fransozo \& EE Boschi. 2007. Reproductive pattern of the South American endemic shrimp Artemesia longinaris (Decapoda: Penaeoidea), off São Paulo State, Brazil. Revista de Biología Tropical 55(1): 39-48.

De la Garza J. 2003. Relaciones morfométricas del langostino patagónico (Pleoticus muelleri). Informe Técnico Interno INIDEP 101: 1-9.

Díaz GA, SG Smith, JE Serafy \& JS Ault. 2001. Allometry of the growth of pink shrimp Farfantepenaeus duorarum in a subtropical bay. Transactions of the American Fisheries Society 130: 328-335.

Díaz AC, AM Petriella \& JL Fenucci. 2003. Ciclo de muda y reproducción de la población de langostino Pleoticus muelleri (Crustacea, Penaeoidea) de Mar del Plata. Ciencias Marinas 29(3): 343-355.

Fernández M. 2005. Localización de las concentraciones reproductivas del langostino en el litoral patagónico. Periodo analizado: Diciembre de 2000 a enero de 2005. Informe Técnico Interno INIDEP 77: 1-13.

Fernández M \& D Hernández. 2002. Crecimiento relativo del langostino Pleoticus muelleri (Bate, 1888) del golfo San Jorge, Argentina. Boletín Científico 7: 95-120.

Fernández M, J de la Garza \& G Macchi. 2002. Estimación de la fecundidad potencial y fecundidad relativa del langostino del Golfo San Jorge y litoral de la provincia del Chubut. Periodo analizado: Septiembre 2001 a febrero de 2002. Informe Técnico Interno INIDEP 36: 1-34.

Fernández M, D Hernández \& A Roux. 2007. Distribución espacial del langostino patagónico (Pleoticus muelleri, Bate, 1888)) y su relación con las variables ambientales, Golfo San Jorge, Argentina. Revista de Biología Marina y Oceanografía 42(3): 335-344.

Fischbach C. 1993. Ecología larval del langostino (Pleoticus muelleri). Algunos aspectos de la dinámica de los estadios planctónicos en el litoral bonaerense (Argentina). Frente Marítimo 14(A): 101-109.

Harán NS, JL Fenucci \& AC Díaz. 1992. Efectos de la temperatura y salinidad sobre el crecimiento y la supervivencia del camarón (Artemesia longinaris) y del langostino (Pleoticus muelleri). Frente Marítimo 11(A): 7983. 
Iorio MI, GJ Macchi, CE Fischbach \& HE Christiansen.

1996. Estudios sobre la dinámica reproductiva del langostino (Pleoticus muelleri) en el área de Bahía Blanca (Provincia de Buenos Aires, Republica Argentina). Frente Marítimo 16(A): 111-118.

Iorio MI, GJ Macchi \& D Hernández. 2000. Estimación de la talla de primera madurez y fecundidad del langostino patagónico. Caracterización del desarrollo del ovario y el estadio de impregnación. Informe Técnico Interno INIDEP 61: 1-14.
Macchi GJ, MI Iorio \& A Aubone. 1998. Estimación de la fecundidad del langostino argentino Pleoticus muelleri Bate, 1888 de Patagonia (sur de Argentina). Boletín del Instituto Español de Oceanografía 14(1/2): 19-29.

SAGPyA 2008. República Argentina Exportaciones e Importaciones Pesqueras 2007, 74 pp. Secretaría de Agricultura, Ganadería, Pesca y Alimentos, Buenos Aires.

Recibido el 8 de enero de 2008 y aceptado el 1 de octubre de 2008 